\title{
Mast cells evaluation in meningioma of various grades
}

\author{
Joanna Reszec ${ }^{1}$, Adam Hermanowicz ${ }^{2}$, Jan Kochanowicz ${ }^{2}$, \\ Grzegorz Turek², Zenon Mariak², Lech Chyczewski ${ }^{1}$ \\ ${ }^{1}$ Department of Medical Pathomorphology Medical University of Bialystok \\ ${ }^{2}$ Department of Neurosurgery Medical University of Bialystok
}

\begin{abstract}
Meningioma is a heterogenous group of primary brain tumors. The progression or recurrence is relatively very common; however, prognostic factors which may indicate those events are not known. The aim of the study was to evaluate the presence of mast cells within the low grade and high grade meningiomas. The material included 70 cases of meningomas ( $63 \mathrm{G1}$ grade cases) of adult subjects (range 23-84 years). In paraffin sections presence of tryptase, a marker of mast cells, was detected by immunohistochemistry in 10 random fields in each slide under the light microscope. The presence of the peritumoral oedema was estimated by brain computer tomography. The expression of tryptase was observed in $32 \%$ of low grade meningiomas and $86 \%$ of high grade meningiomas. The immunostained cells were observed close to the blood vessels. We conclude that the number of mast cells might be a significant prognostic factor for the recurrence or bad prognosis of meningiomas. (Folia Histochemica et Cytobiologica 2012, Vol. 50, No. 4, 542-546)
\end{abstract}

Key words: meningioma, mast cell, tryptase, immunohistochemistry, peritumoral edema, CT

\section{Introduction}

Meningiomas are relatively common, slowly growing primary intracranial brain tumors, typically arising from the arachnoidal cap cell of the meninges. The etiology is still unclear; however there is a close association with the brain trauma or previous head and neck radiation. Also female gender commonly predisposes to meningioma development, probably due to the progesterone action [1]. According to WHO classification meningiomas are graded as G1 (meningothelial, transitional, secretory, fibrous, psammomatous, etc), G2 (atypical, clear cell or chordoid) or G3 (anaplastic, papillary or rhabdoid). Though this classification involves the presence of such histological features like necrosis, mitotic figures, or nuclear polymorphism [2], tumors belonging to the same subgroups can show quite different biological character-

Correspondence address: J. Reszec, Department of Medical Pathomorphology Medical University of Bialystok Waszyngtona St. 13, 15-269 Bialystok, Poland; e-mail: joasia@umwb.edu.pl istics like local invasiveness and growth rate, predisposition to local recurrence after surgical excision, predisposition to malignancy, presence of peritumoral brain edema, etc. All these differences cannot be explained by known molecular aspects of the meningioma development which mostly correlate with NF2 gene mutation (deletion in 22 pair of chromosom $22 q 12)$, resulting in merlin protein inactivation. The frequency of NF2 mutation is similar both in welldifferentiated and in atypical or anaplastic meningiomas, therefore NF2 is a very important factor in the meningioma development, however, not in tumor progression [3]. The prognosis in most cases is good and surgical treatment is curable; however, in some instances recurrence or even progression into less differentiated meningioma is observed. Still there is lack of the prognostic factors, which allow predicting those cases which might behave more aggressively.

Mast cells (MC) are among agents of known potential for promotion of neoplastic transformation. They contain mediators that can affect fibroblasts or other inflammatory cells and secrete various cytokines and other vasoactive/inflammatory substances or growth factors [5-8]. MC are derived from CD34 
positive hematopoietic precursors cells residing in the bone marrow. They mature in different tissues depending on microenvironmental conditions and play an important role in allergic reactions, inflammation or autoimmunological processes [4]. They can be found also in the central nervous system where they encircle endothelial cells and pericytes [2]. Brain mast cells increase in number during neonatal development or in association with neuronal injury. It is considered that mast cells may promote development of tumors through many pathways. Angiogenesis can be promoted by the activation of endothelin 1 and 2 and/ /or by activation of different growth factors, like Vascular Endothelial Growth Factor (VEGF), IL-8, Epidermal Growth Factor (EGF), Fibroblast Growth Factor-2 (FGF-2) and others. High mast cells content is found in neurofibromas, malignant schwannomas, leiomyosarcomas or in other malignancies. Recently it was found that mast cells activation facilitates metastases to the brain as well as progression of breast or lung cancer. This is due to the activation of histamine, which through $\mathrm{H} 1$ receptor induces tumor cell proliferation. Moreover, activation of metalloproteinases disturbs the normal stroma-epithelium communication and permits tumor invasion $[2,5,9]$.

The aim of the study was to determine the presence of mast cells in the meningiomas of various grades.

\section{Material and methods}

We examined specimens of 70 meningiomas surgically removed in the Department of Neurosurgery, Medical University of Bialystok in years 2000-2011. The specimens were fixed in $10 \%$ buffered formalin, and then routinely embedded in paraffin blocks. Slides were stained with hematoxylin and eosin and evaluated by pathologist as meningiomas G1 (low grade meningiomas) and meningiomas G2 or G3 (high grade meningiomas). We also estimated the presence of the peritumoral oedema in all examined cases. Immunohistochemical reaction was carried out to estimate the presence of mast cells. Following the deparaffinision and rehydration, epitope retrieval was performed in the EnVision Flex Target Retrieval Solution (DAKO) in high pH. Endogenous peroxidases were blocked by incubating the sections in methanol and 30\% hydrogen peroxydase for 20 minutes. Next, slides were incubated with a typical mast cells' marker FLEX Monoclonal Mouse Anti-Human Mast Cell Tryptase, (clon AA1), Ready-to-Use (Link) in room temperature for 20 minutes, complexes were visualized with EnVision FLEX, High pH (Link) (DAKO) and with DAB for 10 minutes.

Appropriate positive and negative controls were performed. Negative controls were performed using a non-immunized IgG replacing the primary antibody. We used sections of skin mastocytosis patient as a positive control.

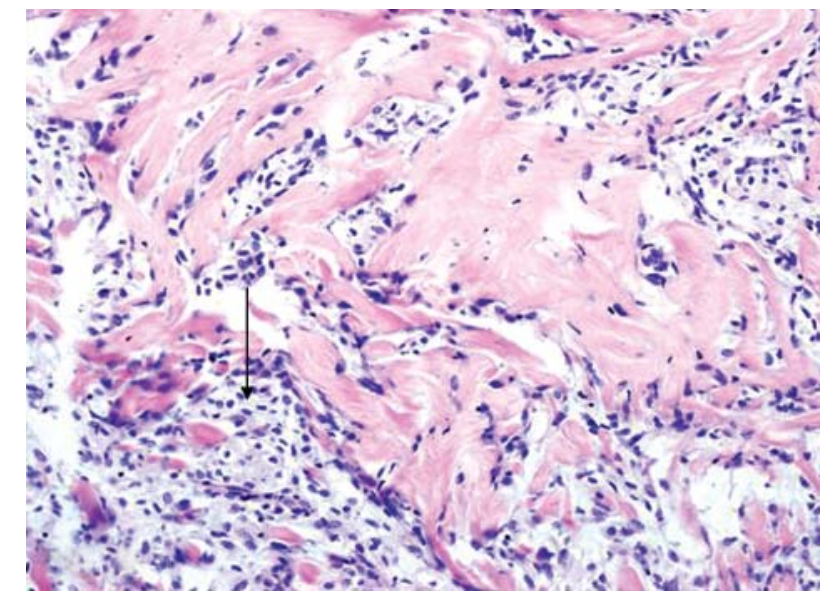

Figure 1. Low grade meningioma with the infiltration of the dura matter, but without nuclear polymorphism or high index of mitotic figures; $100 \times$

The slides were then counterstained with hematoxylin and examined under the light microscope Olympus BX45. Immunohistochemical evaluation of tryptase expression was performed by two independent pathologists. The results were expressed as the percentage of mast cells with a strong positive tryptase staining and labeled as follows: negative (-) with $\leq 10 \%$ of positive cells, positive $(+)$ with $11 \%$ to $50 \%$ of positive cells and highly positive $(++)$ with $\geq 51 \%$ positive cells in a set of random 10 fields under $20 \times$ magnification.

Statistical analyses were performed by the use of $\chi^{2}$ test and Pearson's correlation (Statistica 8,0 software). Values of $\mathrm{p}<0,05$ were considered as statistically significant.

\section{Results}

Among 70 specimens of meningioma there were 63 meningiomas G1: 33 out of them were estimated as transitional (Figure 1), 10 as meningothelial, 12 as fibrous (Figure 2) and 8 as mixed meningioma: mostly psammomatous -5 and secretory -3 . The remaining meningiomas were in 5 cases atypical (G2) and in 2 cases anaplastic (G3) (Figure 3 and 4 respectively). This material originated from 23 male and 47 female patients. Among 7 cases of atypical and anaplastic meningiomas there was only 1 female patient and 6 were males. The age of patients in the G1 group (I group - low grade) ranged from 28 to 84 years. In the $\mathrm{G} 2$ and $\mathrm{G} 3$ group (II group - high grade) the age ranged from 26 to 43 years.

The expression of tryptase in low and high grade meningiomas is summarized in Table 1. In the I group (low grade meningiomas) mast cells were observed in $20 / 63$ cases $(31,8 \%)$, however $8 / 20$ cases presented a strong diffused immunoreaction (Figure 5). It was of note that all psammomatous, secretory and men- 


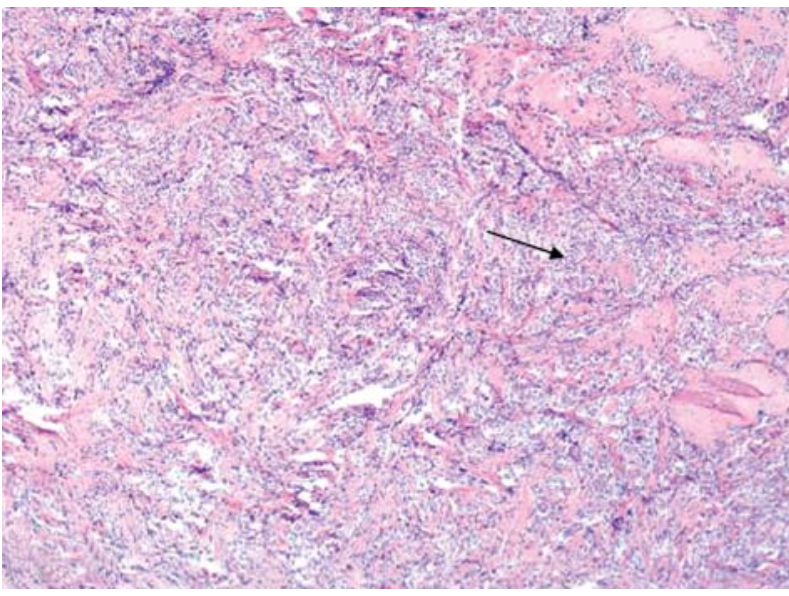

Figure 2. Fibrous low grade meningioma with hyalinization fields (histopathological slide for the Fig. 1); $40 \times$

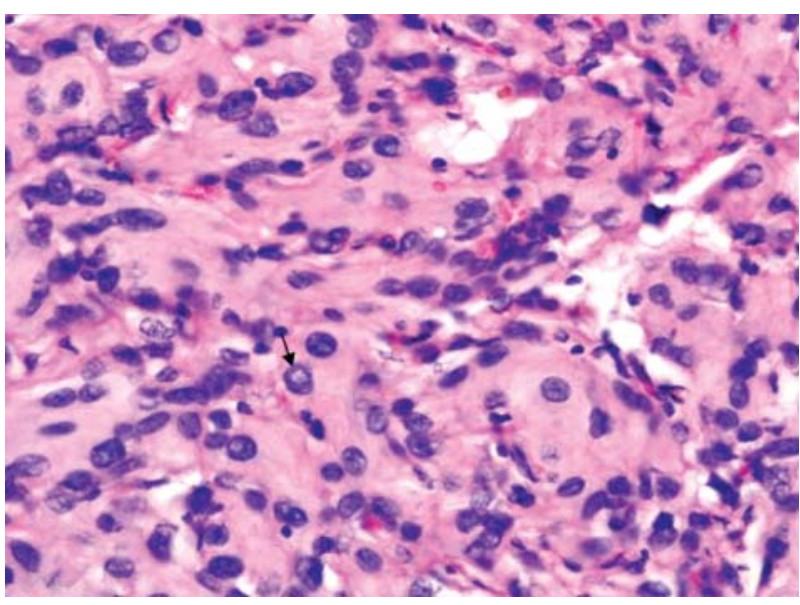

Figure 3. Atypical (high grade) meningioma with the nuclear polymorphism. The arrow shows intranuclear inclusions typical for meningioma (mitotic figures $>4 / 10 \mathrm{hpf}$ ); $400 \times$

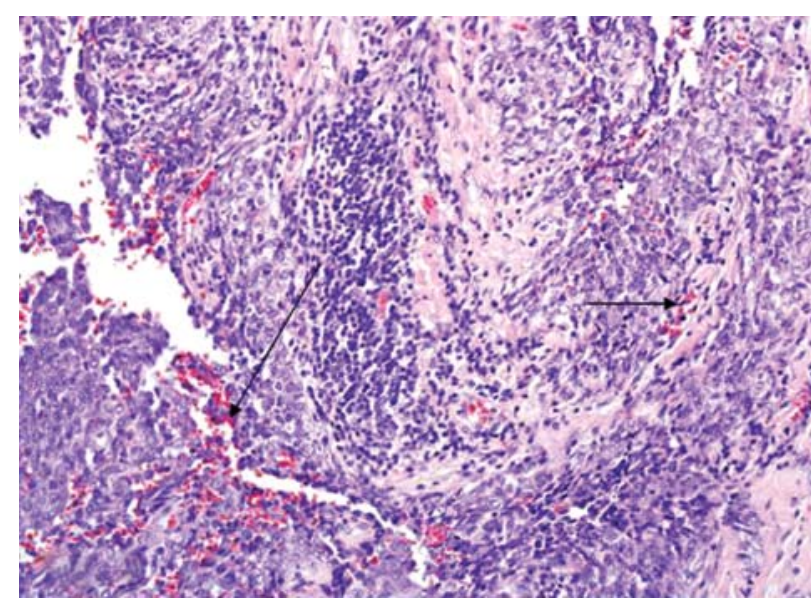

Figure 4. High grade (anaplastic) meningioma with marked inflammatory cells infiltration (histopathological slide for the Fig. 3); $100 \times$
Table 1. Tryptase expression in low and high grade meningiomas

\begin{tabular}{|l|c|c|c|c|c|c|}
\hline \multirow{2}{*}{$\begin{array}{l}\text { Tryptase } \\
\text { expression }\end{array}$} & \multicolumn{2}{|c|}{ Negative } & \multicolumn{2}{c|}{$(+)$ positive } & \multicolumn{2}{c|}{$(++)$ positive } \\
\cline { 2 - 7 } & No & $\%$ & No & $\%$ & No & $\%$ \\
\hline $\begin{array}{l}\text { Low grade } \\
\text { (I group) }\end{array}$ & 43 & $68,2 \%$ & 12 & $19 \%$ & 8 & 12,8 \\
\hline $\begin{array}{l}\text { High grade } \\
\text { (II group) }\end{array}$ & 1 & $14,3 \%$ & 5 & $71,4 \%$ & 1 & $14,3 \%$ \\
\hline
\end{tabular}

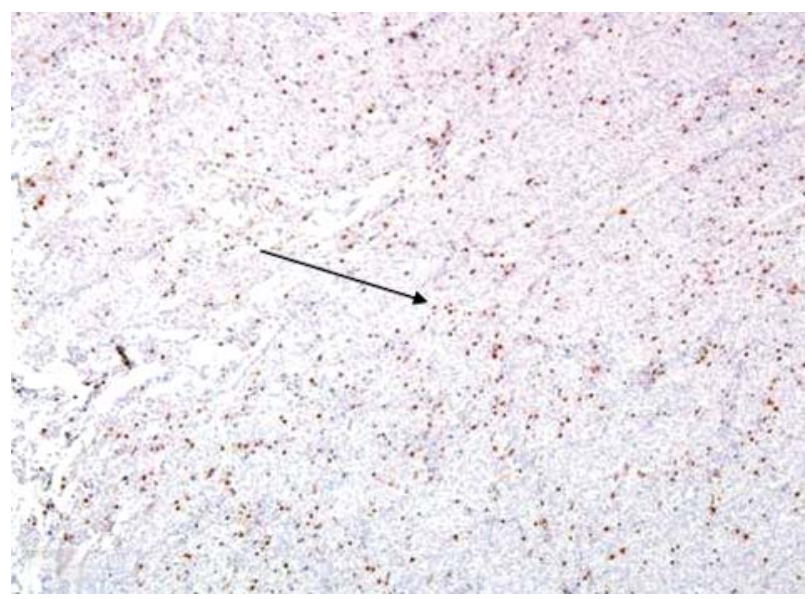

Figure 5. Strong diffuse tryptase expresion within meningioma of low grade maliganacy; $40 \times$

ingothelial meningiomas were negative for tryptase, whereas all 12 fibrous and 8 transitional meningiomas were positive for tryptase. Comparing all tryptasepositive high grade meningiomas we found that by CT brain examination all were sourrounded by marked peritumoral edema. The immunostaining was observed mostly next to blood vessels disseminated within the tumor.

In the group II (high grade meningiomas) all of the tumors presented edematous changes in CT and only $1 / 7$ cases was negative for tryptase. One anaplastic meningioma (Figure 6) presented a strong diffused immunoreaction, the rest of the high grade meningiomas showed focal but disseminated positive immunoreaction for tryptase (Figures 7, 8).

\section{Discussion}

Although most meningiomas are benign, they have a broad spectrum of clinical and histological pattern, especially a high incidence of recurrence, even those estimated as low grade meningiomas after total excision. Histopathologically there are many types of meningiomas, but the most important features are the 


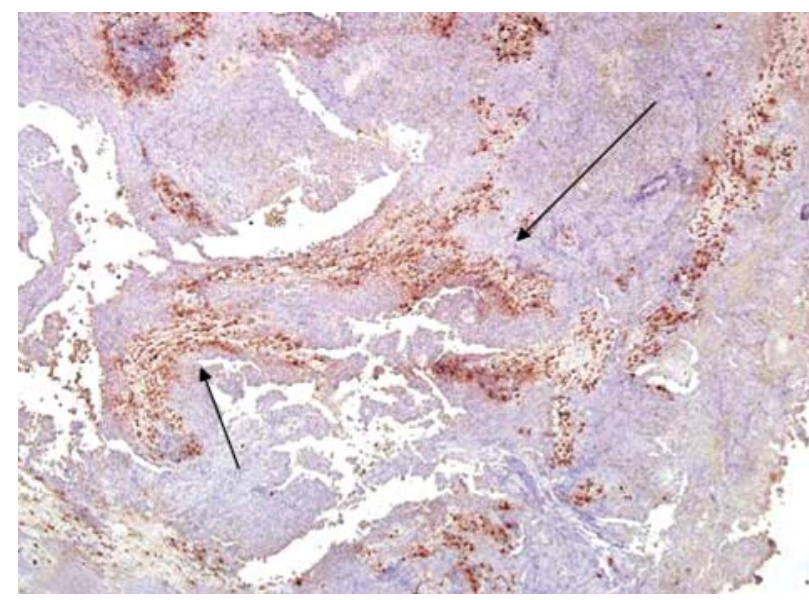

Figure 6. Strong diffuse tryptase immunoexpression within high grade (anaplastic) meningioma infiltrating the skull; $40 \times$

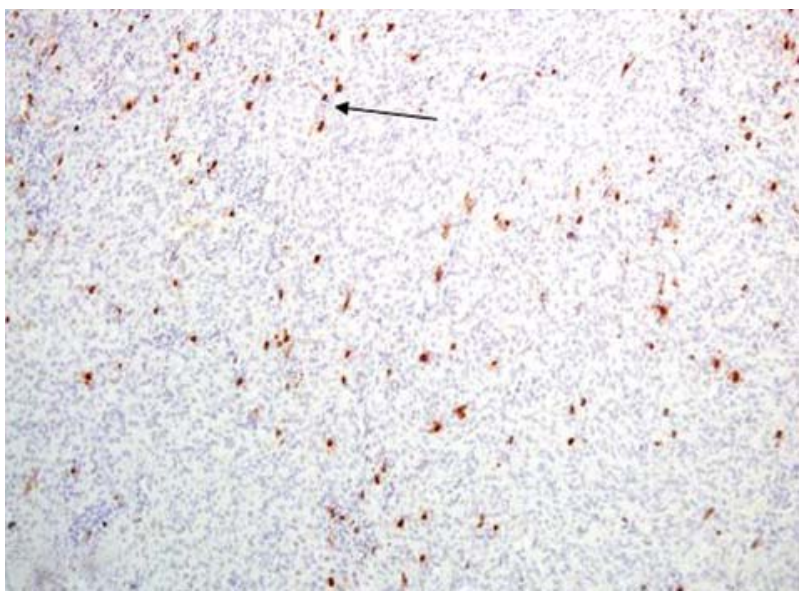

Figure 7. Tryptase expression in high grade meningioma (atypical) with marked peritumoral edema; $100 \times$

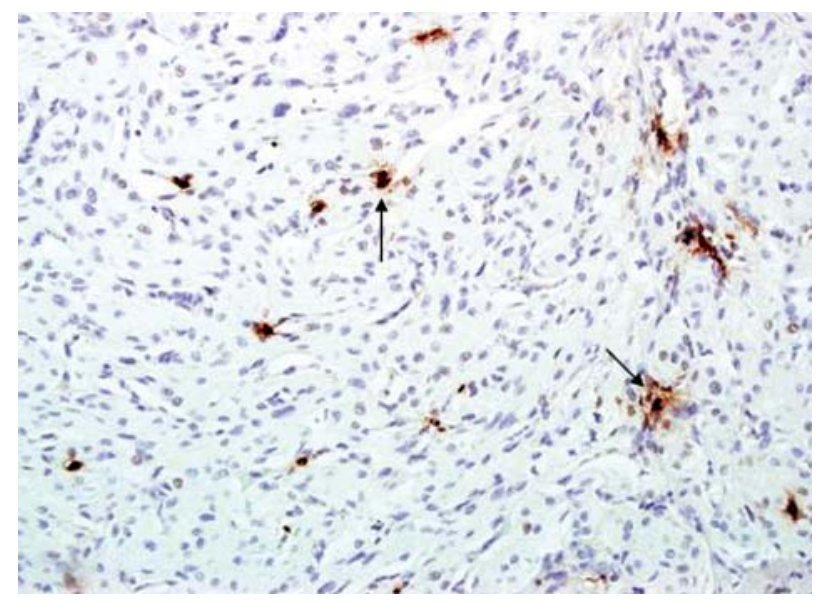

Figure 8. Few tryptase positive mast cells within the G1 meningioma with marked brain edema; $200 \times$ number of mitotic figures, the presence of necrosis and the nuclear polymorphism. However, sometimes these features are not useful enough to predict the tumors progression; that is why other prognostic factors are important to estimate. Some data indicate that not only genetic alterations but also expression of growth factors (like VEGF or PDGF) can predict tumor progression [5, 6]. However, so far there is lack of the prognostic factors, which may be associated with the meningioma progression or recurrence. In this study we estimated the presence of mast cells within the meningioma of various grades.

We found that in high grade meningiomas the tryptase expression was very strong and diffused; however, also in low grade meningiomas we observed the presence of mast cells especially next to the blood vessels. It is of note that in slow growing psammomatous and meningothelial meningiomas no tryptase expression was demonstrated. This may be due to the suppressive effect of psammoma bodies on tumor growth or due to the effect of tumor environment in psammoma formation [10]. There is little data concerning the presence of mast cells in meningiomas. Moradi et al. [2] observed no correlation between the count of mast cells and the grade of meningiomas, a finding which is in opposition to our results. On the other hand Theoharides et al. [9] suggested that inflammation including mast cells accumulating around the tumor could promote tumor growth. Also Tirakotai et al. [11] demonstrated a high number of mast cells in secretory meningiomas and found a correlation with peritumoral oedema. In our study we found an association between the presence of mast cells and brain oedema, especially in high grade group, however, there was no statistically significant correlation between both parameters because of low number of examined cases. Epari et al. [12] and Tena-Suck et al. [13] observed a significant number of mast cells and B lymphocytes in chordoid meningioma, in the stroma of connective tissue. Similar to our results were obtained by Bo et al. [14] who observed a statistically significant association between the number of macrophages and mast cells and microscopic features of aggressiveness, i.e. high cellularity as well as the combination of nuclear atypia and frequent mitotic figures. Also Schober et al. [15] observed in meningiomas mast cells and microcystic alterations which associated with local vascular permeability disturbances. Popovic et al. [16] presented a case of meningioma containing a unusually high number of mast cells and suggested the relationship between such subtype of meningioma and subdural hematoma.

In many types of malignant tumors the number of infiltrating mast cells was shown to correlate with the 
clinical features and to represent a significant prognostic factor. That progressive increase in mast cell number associated with an increasingly poor prognosis has been shown during the progression of human melanoma and oral squamous carcinoma [2, 4].

Mast cells synthesize and store large amounts of metalloproteinases (MMP-2, MMP-9) and serine-proteinases of two subclasses, i.e. tryptase and chymase. Given the ability of MMP-2 and MMP-9 to degrade type IV, V, and VII collagens and fibronectin, the major components of the interstitial stroma and subendothelial basement membrane, mast cells may contribute to the in situ progression of invasive and metastatic solid tumors $[6,9,17]$. Our results suggest that the number of mast cells in meningioma might be a prognostic factor for bad prognosis and tumors recurrence.

\section{References}

1. Riemenschneider MJ, Perry A, Reifenberger G Histological classification and molecular genetics of meningiomas. Lancet Neurol. 2006;5:1045-1054.

2. Moradi A, Semnani V, Djam H, Tajodini A, Zali AR, Ghaemi K, Nikzad N, Madani-Civi M. Pathodiagnostic parameters for meningioma grading. J Clin Neurosci. 2008;15:1370-1375.

3. Heinrich B, Hartmann C, Stemmer-Rachamimov AO, Louis DN, MacCollin M. Multiple meningiomas: Investigating the molecular basis of sporadic and familial forms. Int J Cancer. 2003;103:483-488.

4. Maltby S, Khazaie K, McNagny KM. Mast cells in tumor growth: angiogenesis, tissue remodelling and immune-modulation. Biochim Biophys Acta. 2009;1796:19-26.

5. Ribatti D, Crivellato E. Mast cells, angiogenesis and cancer. Adv Exp Med Biol. 2011;716:270-288.

6. Ribatti D, Crivellato E. Mast cells, angiogenesis, and tumour growth. Biochim Biophys Acta. 2012;1822:2-8.
7. Nautiyal KM, Ribeiro AC, Pfaff DW, Silver R. Brain mast cells link the immune system to anxiety-like behavior. Proc Natl Acad Sci USA. 2008;105:18053-18057.

8. Jin Y, Silverman AJ, Vannucci SJ. Mast cells are early responders after hypoxia-ischemia in immature rat brain. Stroke. 2009;40:3107-3112.

9. Theoharides TC, Rozniecki JJ, Sahagian G, Jocobson S, Kempuraj D, Conti P, Kalogeromitros D. Impact of stress and mast cells on brain metastases. J Neuroimmunol. 2008;205:1-7.

10. Das DK. Psammoma body: a product of dystrophic calcification or of a biologically active process that aims at limiting the growth and spread of tumor? Diagn Cytopathol. 2009;37:534-541.

11. Tirakotai W, Mennel HD, Celik I, Hellwig D, Bertalanffy H, Riegel T. Secretory meningioma: immunohistochemical findings and evaluation of mast cell infiltration. Neurosurg Rev. 2006;29:41-48.

12. Epari S, Sharma MC, Sarkar C, Garg A, Gupta A, Mehta VS. Chordoid Meningioma, an uncommon variant of meningioma: of 12 cases $J$ Neurooncl. 2006;78:263-269.

13. Tena-Suck ML, Collado-Ortěz MA, Salinas-Lara C, García-López R, Gelista N, Rembao-Bojorquez D. Chordoid meningioma: a report of ten cases. J Neurooncol. 2010;99: 41-48.

14. Bø L, Mørk SJ, Nyland H. An immunohistochemical study of mononuclear cells in meningiomas. Neuropathol Appl Neurobiol. 1992;18:548-558.

15. Schober R, Himuro H, Wechsler W. Cystic changes and vascular permeability in meningiomas. Clin Neuropathol. 1988;7:16-21.

16. Popovic EA, Lyons MK, Scheithauer BW, Marsh WR Mast cell-rich convexity meningioma presenting as chronic subdural hematoma: case report and review of the literature. Surg Neurol. 1994;42:8-13.

17. Crivellato E, Nico B, Ribatti D. Mast cell contribution to tumor angiogenesis: a clinical approach. Eur Cytokine Netw. 2009;20:197-206. 